\title{
Laparoscopic distal pancreatectomy
}

\author{
Bulent Salman ${ }^{1}$, Tonguc Utku Yilmaz ${ }^{2}$, Kursat Dikmen ${ }^{1}$, Mehmet Kaplan $^{3}$ \\ ${ }^{1}$ Department of General Surgery, Gazi University School of Medicine, Ankara, Turkey; ${ }^{2}$ Department of General Surgery, Kocaeli University School \\ of Medicine, Kocaeli, Turkey; ${ }^{3}$ Department of General Surgery, Bahcesehir University School of Medicine, Istanbul, Turkey \\ Contributions: (I) Conception and design: B Salman, TU Yilmaz; (II) Administrative support: All authors; (III) Provision of study materials or patients: \\ K Dikmen, M Kaplan; (IV) Collection and assembly of data: B Salman, TU Yilmaz, K Dikmen; (V) Data analysis and interpretation: M Kaplan; \\ (VI) Manuscript writing: TU Yilmaz, B Salman; (VII) Final approval of manuscript: All authors. \\ Correspondence to: Bulent Salman, MD, FACS. Associated Professor, Department of General Surgery, Gazi University School of Medicine, C Block, \\ 7.Floor, Emniyet Mahallesi, Besevler, 06500, Ankara, Turkey. Email: bsalman@gazi.edu.tr; bulentsalman@gmail.com.
}

\begin{abstract}
After technological advances and increased experiences, more complicated surgeries including distal pancreatectomy can be easily performed with acceptable oncologic results, and decreased mortality and morbidity. Laparoscopic distal pancreatectomy (LDP) has been shown to have several advantages including less blood loss, less hospital stay, less pain. Several studies comparing open distal pancreatectomy (ODP) and LDP resulted that both techniques have similar results according to pancreas fistulas, oncological results, costs and operation indications. Morbidity is very low in high volume centers, for this reason at least ten cases should be performed for the learning curve. Several authors remarked important technical points in LDP in order to perform safe and acceptable LDP in several studies. Here in this review, we aimed to overview the results of previous studies about LDP and discuss the technical points of LDP.
\end{abstract}

Keywords: Pancreas; distal pancreatectomy; laparoscopic; pancreatic resection

Received: 18 June 2016; Accepted: 18 July 2016; Published: 12 August 2016.

doi: 10.21037/jovs.2016.07.21

View this article at: http://dx.doi.org/10.21037/jovs.2016.07.21

\section{Introduction}

Despite the developments in minimally invasive surgery for intraabdominal pathologies, laparoscopic pancreatic surgery has lagged behind because of its limitations, such as major vascular proximity, retroperitoneal location, and adjacent organs (1). However, with improvements in laparoscopic skills and surgical technology, laparoscopic distal pancreatectomy (LDP) has been proven to be safe and have better outcomes (2). In the present study, we compared LDP with the traditional method of open distal pancreatectomy (ODP).

Early series of LDP consisted of benign lesions, such as premalignant lesions, benign pancreatic strictures, and neuroendocrine pancreatic lesions (3). In these early series, LDP reduced postoperative morbidity and hospital stay and increased quality of life in young patients. Then, the number of LDP procedures performed for malignant disease increased $(2,4)$. It has been shown in several studies that tumors can be resected with adequate lymph nodes using LDP, with similar pancreatic fistula rates (4). On the other hand, technically demanding spleen-preserving distal pancreatectomies have been performed (5). In this article, we describe current concepts of LDP.

\section{Indications}

Symptomatic benign lesions, premalignant lesions, and cancer located in the body or tail of the pancreas are candidates for distal pancreatectomy. Until recently, the number of distal pancreatectomy procedures performed was limited because of the low incidence of pancreatic lesions and the high proportion of lesions unresectable at first presentation. Today, however, new diagnostic tests are available that are capable of providing an early diagnosis of pancreatic lesions with high quality, and the number of indications for distal pancreatectomy has increased. Since the first series of LDP cases published in 1996, the benefits and safety of LDP have been proven $(4,6)$. During preoperative assessment, medical 
comorbidities, tumor size, organ involvement, and major vascular involvement are evaluated.

Recent studies show that there is no absolute indication about how the type of surgery (open or laparoscopic) is decided. Consideration of individual patients' features to decide on the type of operation has been reported in several studies (7-11). There were no differences between LDP and ODP cases in terms of patient age, gender, American Society of Anesthesiologists score, body mass index (BMI), or presence of diabetes mellitus (7-11). Cho et al. showed that no preoperative evaluable variables were associated with a higher likelihood of significant fistula after LDP $v s$. ODP (12). Greater BMI, larger specimen size, and increased blood loss were much more important risk predictors for postoperative complications after ODP as compared with LDP (12).

In selected studies, the ratios of ODP and LDP were $14.2 \%$ and $8.8 \%$ for endocrine tumors, $16.8 \%$ and $9.7 \%$ for mucinous cystic neoplasias, $9.7 \%$ and $6.7 \%$ for chronic pancreatitis, $7 \%$ and $8 \%$ for pseudocysts, $8.5 \%$ and $6.2 \%$ for intraductal papillary mucinous neoplasms, $7.8 \%$ and $17.5 \%$ for ductal adenocarcinomas, $7.3 \%$ and $16.5 \%$ for pancreatic tumors, and $16.1 \%$ and $17 \%$ for cystic lesions, respectively. These results show that LDP and ODP have been performed in similar ratios for cystic lesions, chronic pancreatitis, intraductal papillary mucinous neoplasms, and pseudocysts. Ductal adenocarcinomas and pancreatic tumors, however, have been treated more often with ODP. Mucinous cystic neoplasias have been treated much more often with LDP (13). Because of the concern about achieving good oncological outcomes, LDP has been thought to be contraindicated in patients with malignant disease. Trocar site metastasis, promotion of neoplastic growth by pneumoperitoneum, and wound recurrence have not been proven to be risks of $\operatorname{LDP}(14,15)$. However, no evidence has been presented in the literature that the use of the laparoscopic technique increases the risk of neoplastic dissemination, and none of the patients in recent studies developed any trocar site or peritoneal metastasis (4,16-19). The results described above show that there is no exact preoperative indication for ODP or LDP.

Distal adenocarcinomas of the body and tail of the pancreas comprise only $20-25 \%$ of all diagnosed pancreatic adenocarcinomas, and surgical resection remains the only potentially curative therapy (20). In pancreatic cancer, negative surgical margins and adequate lymph node harvesting are crucial. These factors lead to long survival. To achieve these oncological outcomes, radical antegrade modular pancreatosplenectomy (RAMPS) seems superior to conventional distal pancreatectomy (21). It has been hypothesized that improved oncological resection could be achieved with RAMPS, with a higher likelihood of obtaining negative tangential margins (89\%) and increased rates of R0 resection (81\%) (22). Use of the RAMPS approach can increase R0 rates. The RAMPS technique has been adopted for laparoscopic surgery and is an option for the laparoscopic resection of distal pancreatic adenocarcinomas $(19,23)$. Advanced laparoscopic operations, such as RAMPS can be easily performed. Also, additional organ resections are not contraindications to LDP. Colectomy, gastrectomy, cholecystectomy, and repair of colovesical fistulas have been laparoscopically performed with LDP $(19,24)$.

\section{Surgical techniques}

Since Gagner first described the LDP method, this operative technique has been modified at different centers. LDP is usually performed with the patient supine or on the left side $(25,26)$. The advantages of the supine position are ease of setup, clearer airway access for anesthesia, and ability to access the pancreatic head and neck. Four or five trocars are placed in a semicircular fashion around an umbilical camera. Alternatively, trocar sites are placed under direct visualization, depending on the patient's body habitus and the location of the lesion. A left lateral decubitus position facilitates exposure of the left upper abdominal quadrant (1). A lateral position allows gravity retraction of the stomach and spleen, more direct visualization of the body and tail of the pancreas, and superior ergonomics and comfort for the surgeon (27).

In our practice, the patient is placed supine. The handguided approach has been defined by several authors $(28,29)$. The advantages of the hand-guided approach include preserving the surgeon's ability to perform direct palpation of the tumor and anatomy, ease of removal of larger malignant specimens through the hand port, use of manual dissection, increased surrounding inflammation, improved ability to operate on obese patients, and opportunity to apply direct pressure in case of bleeding. It is not necessary for the surgeon's hand to feel the borders of the lesion. Most authors advocate the use of intraoperative laparoscopic ultrasound to localize the lesion and define the extent of the resection (30). If the lesion is obvious, dissection is initiated by mobilizing the lower pancreatic margin $2 \mathrm{~cm}$ proximal to the lesion. However, for malignant lesions, a formal left pancreatectomy is performed at the level of next to the junction of the superior mesenteric vein (SMV) and the 
portal vein.

There is controversy about splenectomy in LDP. Some authors believe splenectomy should be performed routinely because splenic artery preservation is hazardous for oncological radicality in distal pancreatectomy (31). In order to ensure extensive resection of lymph nodes located along the splenic artery and splenic hilum, splenectomy with splenic artery resection is advised (32). Distal pancreatectomy with splenic vessel preservation may lead to remnant pancreatic tissue on the splenic vessel, and therefore it is not advised in patients with malignant disease. However, in chronic pancreatitis or benign diseases, the number of cases with spleen preservation is high $(33,34)$. Spleen preservation was shown to be associated with reductions of postoperative infection and length of hospital stay. In benign disease, attempts to preserve the spleen are important. In cases of chronic pancreatitis, however, pancreatic calcification, marked edema and fibrosis may occur in splenic vessels, and splenic vessel preservation in those cases may not be possible.

The Warshaw technique has been used with LDP to resect and preserve the spleen (35). It is unclear whether the Kimura or the Warshaw technique is superior. However, spleen-related complications are seen much more often after use of the Warshaw technique than with the Kimura technique, such as postoperative splenectomy ( $2 \% v s .0 \%$, respectively), splenic infarction ( $20.8 \%$ vs. $2 \%$, respectively), and chronic abdominal pain (38\% vs. $0 \%$, respectively) $(36,37)$. Symptomatic splenic infarctions have been reported to be significantly less common after vessel-preserving splenectomies. Interestingly, none of these infarcts evolved to an abscess, and they were all conservatively treated (37). In the study of Baldwin et al., only four patients treated with splenic vessel ligation developed symptomatic splenic infarcts, and three patients underwent splenectomy (38). Patients were routinely monitored postoperatively with computed tomography (CT) in only a limited number of previous studies. For this reason, the number of splenic infarcts might be underestimated. Also, the patients in the Baldwin et al. study were elderly. It is possible that short gastric vessels do not supply enough collateral circulation to support the splenic mass (38). Moreover, supplying enough blood to the spleen might be difficult in patients with large spleens. Thus, it is of paramount importance to evaluate the spleen's dimensions during surgery when deciding on the type of operation to perform.

During spleen-preserving procedures, the spleen should be checked for extensive splenic ischemia, which may be seen in
$10 \%$ of patients. Splenic infarction is seen mostly in the early period. It is reasonable to prefer a distal pancreatectomy with splenectomy to splenic vessel ligation when vessel preservation fails intraoperatively. Perigastric varices and related gastric mucosal bleeding are risks after distal pancreatectomy. In the study of Hwang et al., four patients treated with distal pancreatectomy with splenic vessel ligation had perigastric varices, and only three patients developed submucosal varices (39). Butturini et al. reported perigastric varices in $60 \%$ of patients treated with splenic vessel ligation and $22 \%$ of patients treated with splenic vessel preservation (40); however, bleeding was not seen.

Thus, perigastric varices are not a risk after splenic vessel ligation. The Kimura technique is more demanding, as the splenic vessels are preserved. LDP with splenic vessel preservation is much more time-consuming. However, authors of several systemic reviews have shown that spleen-preserving LDP is much more preferred $(7,12)$. The enhanced surgical view during laparoscopic surgery, with better visualization of splenic vessels, has contributed to these findings. Only 14 patients were converted from vessel preservation to vessel ligation in one study (37). The presence of small breakage of tributary vessels from splenic vessels could potentially obscure the surgical field and result in intraoperative bleeding, splenectomy, or conversion. In spleen-preserving LDP, the pancreas is separated from the splenic vessels. However, this maneuver might be bloody, and it is difficult to manipulate the pancreas.

Velanovich has described the lasso technique, in which a Penrose drain around the neck of the pancreas is used to manipulate the dissection (41). LDP with splenic vessel ligation is $27 \mathrm{~min}$ shorter than LDP with splenic vessel preservation. Eom et al. reported significantly prolonged operative time in spleen preservation compared with splenectomy (194 vs. $251 \mathrm{~min} ; \mathrm{P}=0.02$ ) (42). The mean operative time for LDP ranges from 156 to $383 \mathrm{~min}$, whereas the mean operative time for ODP ranges from 145 to $330 \mathrm{~min}$. The endpoint showed a nonsignificant extension of $9.21 \mathrm{~min}$ of the operative time (13). Blood loss during splenic preservation is reported to be much more than that in LDP with splenectomy (225 vs. $495 \mathrm{~mL}$ ) (23). However, intraoperative blood loss was reported to be less in LDP than in ODP (13). Spleen preservation is much more timeconsuming, technically more demanding, and leads to much more bleeding in LDP than in ODP. The conversion rate for LDP with splenectomy ranges from $0 \%$ to $43 \%$ and LDP with splenic preservation ranges from $72 \%$ to $100 \%$ (1). With increasing experience and specialized centers, the 


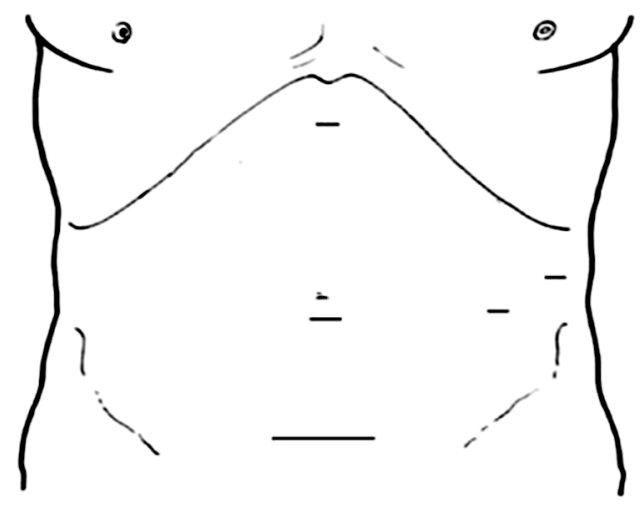

Figure 1 Port placement.

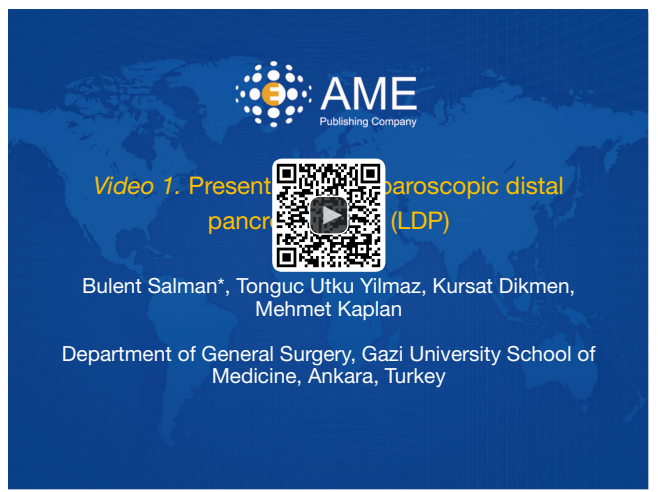

Figure 2 Presentation of laparoscopic distal pancreatectomy (LDP) (43). Available online: http://www.asvide.com/articles/1100

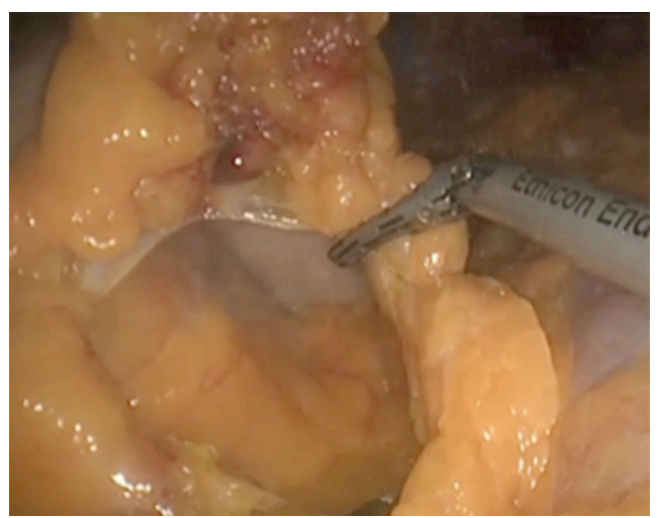

Figure 3 Opening lasser sac.

conversion rates are expected to decrease. The most common reasons for conversion are obesity, dense omental fat, intraoperative bleeding, malignant disease requiring lymph node dissection, inability to detect the tumor, bulky tumors, and peritoneal adhesions due to previous surgery (30).

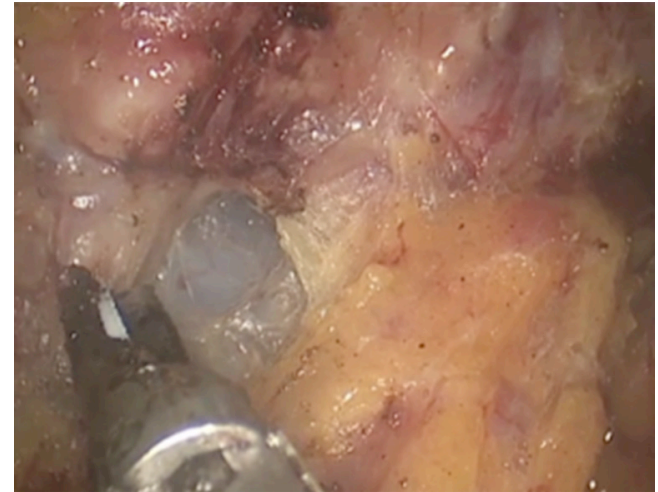

Figure 4 Identification of superior mesenteric vein (SMV).

The patient should be positioned with legs apart or in the left lateral or supine position. The surgeon stands between the patients' legs. An assistant stands on the left side of the patient for camera and a scrub nurse stands on the opposite side. A $10-\mathrm{mm}$ trocar is inserted at the umbilicus for use of the $30^{\circ}$ telescope. A second trocar is inserted in the xiphoid area for retraction of the stomach. A third trocar is inserted in the left subcostal area on the midaxillary line and in the left subcostal area to the midclavicular line (Figure 1).

The patient is placed in a reverse Trendelenburg position to facilitate displacement of the transverse colon and small bowel from the operative field. Video presentation of one of LDPs is given (Figure 2). The patient has a mass with irregular borders in the tail of the pancreas. The lesser sac is opened using an energy device through the avascular plane while preserving the gastroepiploic vessels (Figure 3). Short gastric vessels are dissected to the superior part of the stomach as far as possible. The stomach is grasped and elevated with a nontraumatic grasper introduced through the xiphoid port to enable investigation to the entire neck, body, and tail of the pancreas.

At this step, we routinely use intraoperative laparoscopic ultrasonography to identify the precise location of the tumor and its relation to the splenic vessels and to demarcate its extent. We routinely explore SMV at the inferior border of the pancreas for resectability before starting the dissection. This maneuver requires finding SMV and developing a space between the pancreatic neck and vein. SMV is then readily identified at the inferior margin of the pancreatic neck with a blunt dissector (Figure 4). If there is no invasion, a tunnel is developed easily between the pancreatic neck and splenic vein. Next, the pancreas is hanged with nylon tape for manipulation. After determining resectability, deciding on the dissection begins at SMV and is carried 


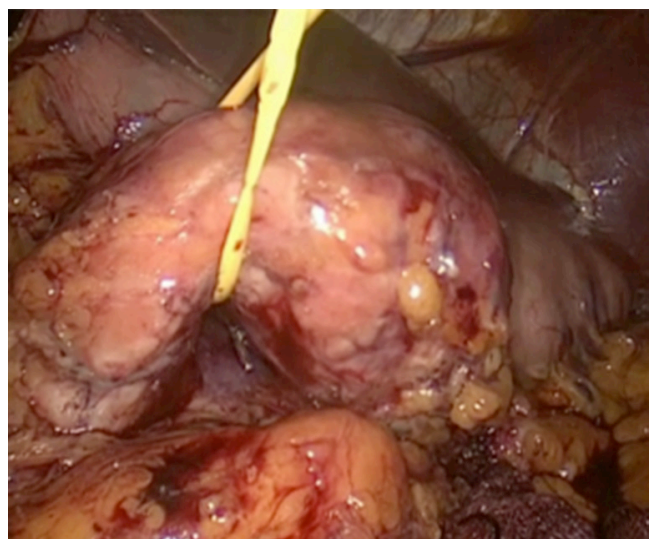

Figure 5 Hanging pancreas.

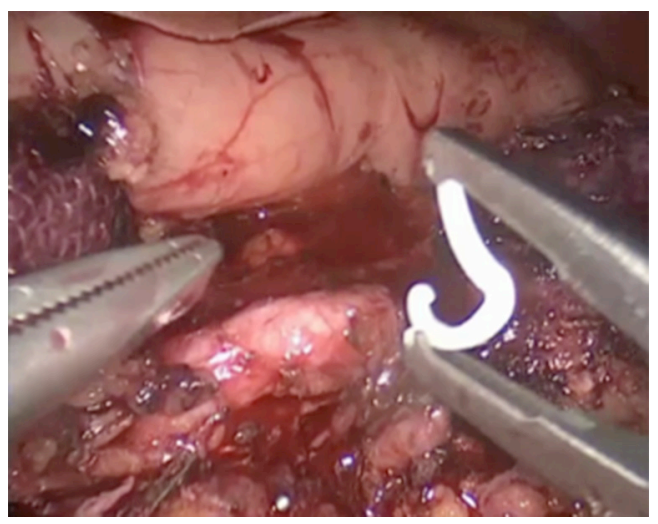

Figure 6 Division of the splenic artery.

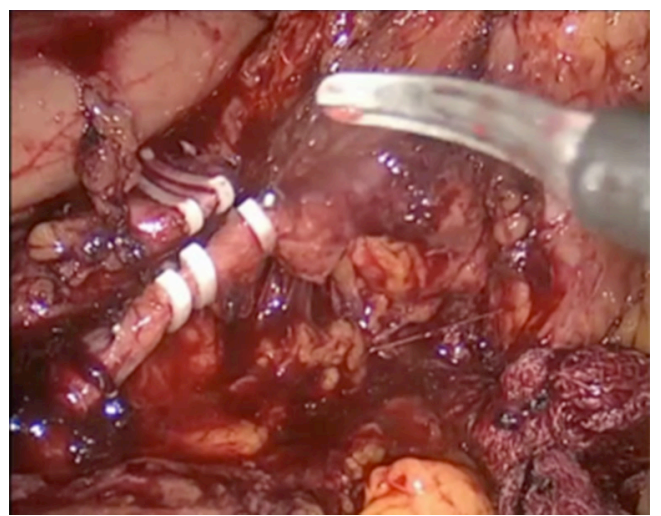

Figure 7 Division of the splenic vein.

laterally along the inferior border of the pancreas, allowing elevation of the posterior margin of the pancreas out of the retroperitoneum (Figure 5).

The splenic flexure of the colon must be mobilized so that the colon does not require continuous retraction to expose the pancreas. At this step, the venous mesentericoportal axis is visualized, and typically, the inferior mesenteric vein can be divided between Weck clips. Then, to start initial mobilization of the spleen, care must be taken regarding progression into the splenic hilum; instead, the dissection should be directed to the inferior pole of the spleen. Complete mobilization requires division of the lateral colon attachments. The splenic artery is controlled on the superior border of the pancreas. Retracting the pancreas inferiorly and laterally reveals the splenic artery and celiac truncus. Circumferential dissection is achieved with a blunt dissector, and initially the splenic artery is divided, usually with a vascular load Endo GIA (Covidien Surgical Boulder, CO, USA) or, on occasion, between Weck clips (Figure 6). At our center, we do not use energy devices for dividing the splenic artery at this step. The artery transection precedes transection of the vein to avoid splenic congestion and bleeding from the transected short gastric vessels.

Because of its fragility and close relation to the pancreas, splenic vein dissection is difficult. The splenic vein is dissected circumferentially, with care taken to identify insertion into both the inferior mesenteric vein and the coronary veins. Then the splenic vein is dissected and divided with the vascular Endo GIA, or, on occasion, between Weck clips, after transection of the pancreas (Figure 7).

The splenic vein has multiple braches that drain the body of the posterior pancreas. Therefore, the pancreas neck must be transected before continuing the dissection. To transect the pancreatic neck, the portal vein must be exposed at the superior margin of the pancreas by identifying the hepatic artery. After clearing a space superiorly and inferiorly, an endoscopic linear stapler can be inserted. Selection of the correct stapler cartridge depends on gland thickness. The goal is to avoid fracturing the gland with a staple length that is too short. If necessary, two staplers can be used for transecting the pancreas. After the splenic vessels are divided, the posterior retroperitoneal space can be dissected easily with the pancreas retracted anteriorly (Figure 8). The spleen is mobilized by continuing the posterior dissection laterally, although the most lateral diaphragmatic attachment may require rotating the spleen medially. This dissection is accomplished with energy devices.

The spleen and pancreas are usually detached at the splenic hilum with ultrasonic shears so that the pancreas can be delivered as an intact specimen and the spleen as a morcellated specimen. The specimen is placed in an Endo Catch bag and extracted through a Pfannenstiel incision. 


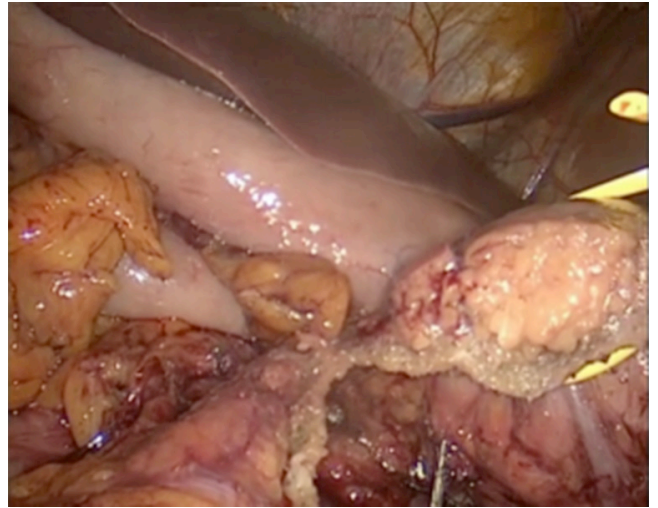

Figure 8 Division of the pancreas.

\section{Pancreatic fistulas}

The most common and clinically relevant complication after distal pancreatectomy is the pancreatic fistula, which may lead to further complications, such as intraabdominal abscess, sepsis, wound infection, delayed gastric emptying, ileus, and lethal bleeding. Treatment of pancreatic fistulas after distal pancreatectomy has not changed for more than 15 years, despite progress in other areas of pancreatic surgery. Several surgical techniques and instruments have been studied with the goal of decreasing pancreatic fistulas. These include hand-sewn sutures, different kinds of staplers, combinations of staplers and sutures, pancreaticojejunal anastomosis, transection by harmonic scalpel, and fibrin glues (44). The experience and results in ODP were the same as those in LDP. Unfortunately, LDP did not decrease the rate of pancreatic fistulas. In a meta-analysis, postoperative pancreatic fistulas were found to occur in $21.7 \%$ of the patients, with no difference between LDP and ODP.

Hand-sewn closures and stapler closures are both used in LDP. As shown in the DISPACT trial, stapler closure is not superior to hand-sewn closure (45). In LDP, stapler closure is the most commonly performed technique. Use of a stapler with 2.5 staple cartridges is associated with fewer pancreatic fistulas than the 4.5 staple cartridges (46). Also, gradual closing of the staple over the course of approximately 2-3 min could reduce the fistula rate (47). In the study by Johnston et al., reinforcement of the staple line with mesh was shown to reduce the fistula rate from $25 \%$ to $10 \%$ (48). Fibrin glue, sealant patches, and seromuscular patches have been used during LDP $(25,49,50)$. However, these modalities were not confirmed in the randomized study of Oláh et al. (51).

As mentioned, LDP can be performed according to oncological principles and with comparably safe procedures.
Shorter hospitalization, less intraoperative blood loss, and decreased pain are advantages of LDP over ODP. On the other hand, cost and the learning period are important factors related to LDP. LDP is a complex abdominal operation and requires experience in laparoscopy. With increasing experience with LDP, operative time is shortening, postoperative pancreatic fistula rate is declining, and operative blood loss is decreasing. Braga et al. found substantial reduction of the conversion rate, operative time, and operative blood loss after experience with the first ten procedures (52). The results of last 20 cases of Braga et al. were similar to the results of high-volume centers (52). The operative time in the learning period was $254 \mathrm{~min}$, but it decreased to $183 \mathrm{~min}$ after the learning period. However, hospitalization time did not show any difference after the learning period. The learning period is usually shorter in high-volume centers than in low-volume hospitals.

Several studies have been performed to compare the costs of LDP and ODP. Korean, Italian, and British studies have shown that LDP is more expensive than ODP $(42,53,54)$. However, decreased length of hospital stay after LDP led to equivalent total hospital costs in the British and Italian studies $(42,53,54)$. In a North American study, overall hospital costs related to LDP were less than those for ODP (55). These studies showed that LDP is a financially reasonable approach to resection. In experienced centers, shorter operative time and decreased complications led to less cost.

\section{Conclusions}

LDP can be safely performed and may produce similar oncological results compared with ODP. Length of hospitalization and intraoperative blood loss in LDP are less when performed at experienced centers. Pancreatic fistula rates are similar with open cases in high-volume centers. Costs of LDP are reasonable in experienced centers. Although LDP surgery is complex, it can be performed safely when standard steps are carefully followed.

\section{Acknowledgements}

None.

\section{Footnote}

Conflicts of Interest: The authors have no conflicts of interest to declare. 


\section{References}

1. Lebedyev A, Zmora O, Kuriansky J, et al. Laparoscopic distal pancreatectomy. Surg Endosc 2004;18:1427-30.

2. Postlewait LM, Kooby DA. Laparoscopic distal pancreatectomy for adenocarcinoma: safe and reasonable? J Gastrointest Oncol 2015;6:406-17.

3. Gagner M, Pomp A, Herrera MF. Early experience with laparoscopic resections of islet cell tumors. Surgery 1996;120:1051-4.

4. Kooby DA, Gillespie T, Bentrem D, et al. Left-sided pancreatectomy: a multicenter comparison of laparoscopic and open approaches. Ann Surg 2008;248:438-46.

5. Bruzoni M, Sasson AR. Open and laparoscopic spleen-preserving, splenic vessel-preserving distal pancreatectomy: indications and outcomes. J Gastrointest Surg 2008;12:1202-6.

6. Mabrut JY, Fernandez-Cruz L, Azagra JS, et al. Laparoscopic pancreatic resection: results of a multicenter European study of 127 patients. Surgery 2005;137:597-605.

7. Jusoh AC, Ammori BJ. Laparoscopic versus open distal pancreatectomy: a systematic review of comparative studies. Surg Endosc 2012;26:904-13.

8. Sui CJ, Li B, Yang JM, et al. Laparoscopic versus open distal pancreatectomy: a meta-analysis. Asian J Surg 2012;35:1-8.

9. Venkat R, Edil BH, Schulick RD, et al. Laparoscopic distal pancreatectomy is associated with significantly less overall morbidity compared to the open technique: a systematic review and meta-analysis. Ann Surg 2012;255:1048-59.

10. Pericleous S, Middleton N, McKay SC, et al. Systematic review and meta-analysis of case-matched studies comparing open and laparoscopic distal pancreatectomy: is it a safe procedure? Pancreas 2012;41:993-1000.

11. Jin T, Altaf K, Xiong JJ, et al. A systematic review and meta-analysis of studies comparing laparoscopic and open distal pancreatectomy. HPB (Oxford) 2012;14:711-24.

12. Cho CS, Kooby DA, Schmidt CM, et al. Laparoscopic versus open left pancreatectomy: can preoperative factors indicate the safer technique? Ann Surg 2011;253:975-80.

13. Mehrabi A, Hafezi M, Arvin J, et al. A systematic review and meta-analysis of laparoscopic versus open distal pancreatectomy for benign and malignant lesions of the pancreas: it's time to randomize. Surgery 2015;157:45-55.

14. Schaeff B, Paolucci V, Thomopoulos J. Port site recurrences after laparoscopic surgery. A review. Dig Surg 1998;15:124-34.

15. Jacquet $\mathrm{P}$, Sugarbaker PH. Wound recurrence after laparoscopic colectomy for cancer. New rationale for intraoperative intraperitoneal chemotherapy. Surg Endosc 1996;10:295-6.

16. Tang CN, Tsui KK, Ha JP, et al. Laparoscopic distal pancreatectomy: a comparative study. Hepatogastroenterology 2007;54:265-71.

17. DiNorcia J, Schrope BA, Lee MK, et al. Laparoscopic distal pancreatectomy offers shorter hospital stays with fewer complications. J Gastrointest Surg 2010;14:1804-12.

18. Kooby DA, Hawkins WG, Schmidt CM, et al. A multicenter analysis of distal pancreatectomy for adenocarcinoma: is laparoscopic resection appropriate? J Am Coll Surg 2010;210:779-85, 786-7.

19. Shin SH, Kim SC, Song KB, et al. A comparative study of laparoscopic vs. open distal pancreatectomy for leftsided ductal adenocarcinoma: a propensity score-matched analysis. J Am Coll Surg 2015;220:177-85.

20. Modolell I, Guarner L, Malagelada JR. Vagaries of clinical presentation of pancreatic and biliary tract cancer. Ann Oncol 1999; 10 Suppl 4:82-4.

21. Strasberg SM, Fields R. Left-sided pancreatic cancer: distal pancreatectomy and its variants: radical antegrade modular pancreatosplenectomy and distal pancreatectomy with celiac axis resection. Cancer J 2012;18:562-70.

22. Mitchem JB, Hamilton N, Gao F, et al. Long-term results of resection of adenocarcinoma of the body and tail of the pancreas using radical antegrade modular pancreatosplenectomy procedure. J Am Coll Surg 2012;214:46-52.

23. Fernández-Cruz L, Cosa R, Blanco L, et al. Curative laparoscopic resection for pancreatic neoplasms: a critical analysis from a single institution. J Gastrointest Surg 2007;11:1607-21; discussion 1621-2.

24. Robinson S, Saif R, Charnley RM, et al. Surgical adjuncts to laparoscopic distal pancreatectomy. Minim Invasive Ther Allied Technol 2011;20:369-73.

25. de Rooij T, Sitarz R, Busch OR, et al. Technical Aspects of Laparoscopic Distal Pancreatectomy for Benign and Malignant Disease: Review of the Literature. Gastroenterol Res Pract 2015;2015:472906.

26. Abu Hilal M, Takhar AS. Laparoscopic left pancreatectomy: current concepts. Pancreatology 2013;13:443-8.

27. Kooby DA. Laparoscopic surgery for cancer: historical, theoretical, and technical considerations. Oncology (Williston Park) 2006;20:917-27; discussion 927-8, 931-2.

28. D'Angelica $M$, Are C, Jarnagin $W$, et al. Initial experience with hand-assisted laparoscopic distal pancreatectomy. 
Surg Endosc 2006;20:142-8.

29. Laxa BU, Carbonell AM 2nd, Cobb WS, et al. Laparoscopic and hand-assisted distal pancreatectomy. Am Surg 2008;74:481-6; discussion 486-7.

30. Abu Hilal M, Jain G, Kasasbeh F, et al. Laparoscopic distal pancreatectomy: critical analysis of preliminary experience from a tertiary referral centre. Surg Endosc 2009;23:2743-7.

31. Andrén-Sandberg A, Wagner M, Tihanyi T, et al. Technical aspects of left-sided pancreatic resection for cancer. Dig Surg 1999;16:305-12.

32. Tol JA, Gouma DJ, Bassi C, et al. Definition of a standard lymphadenectomy in surgery for pancreatic ductal adenocarcinoma: a consensus statement by the International Study Group on Pancreatic Surgery (ISGPS). Surgery 2014;156:591-600.

33. Kiely JM, Nakeeb A, Komorowski RA, et al. Cystic pancreatic neoplasms: enucleate or resect? J Gastrointest Surg 2003;7:890-7.

34. Hutchins RR, Hart RS, Pacifico M, et al. Long-term results of distal pancreatectomy for chronic pancreatitis in 90 patients. Ann Surg 2002;236:612-8.

35. Warshaw AL. Conservation of the spleen with distal pancreatectomy. Arch Surg 1988;123:550-3.

36. Jain G, Chakravartty S, Patel AG. Spleen-preserving distal pancreatectomy with and without splenic vessel ligation: a systematic review. HPB (Oxford) 2013;15:403-10.

37. Partelli S, Cirocchi R, Randolph J, et al. A systematic review and meta-analysis of spleen-preserving distal pancreatectomy with preservation or ligation of the splenic artery and vein. Surgeon 2016;14:109-18.

38. Baldwin KM, Katz SC, Espat NJ, et al. Laparoscopic spleen-preserving distal pancreatectomy in elderly subjects: splenic vessel sacrifice may be associated with a higher rate of splenic infarction. HPB (Oxford) 2011;13:621-5.

39. Hwang HK, Chung YE, Kim KA, et al. Revisiting vascular patency after spleen-preserving laparoscopic distal pancreatectomy with conservation of splenic vessels. Surg Endosc 2012;26:1765-71.

40. Butturini G, Inama M, Malleo G, et al. Perioperative and long-term results of laparoscopic spleen-preserving distal pancreatectomy with or without splenic vessels conservation: a retrospective analysis. J Surg Oncol 2012;105:387-92.

41. Velanovich $\mathrm{V}$. The lasso technique for laparoscopic distal pancreatectomy. Surg Endosc 2006;20:1766-71.

42. Eom BW, Jang JY, Lee SE, et al. Clinical outcomes compared between laparoscopic and open distal pancreatectomy. Surg Endosc 2008;22:1334-8.

43. Salman B, Yilmaz TU, Dikmen K, et al. Presentation of laparoscopic distal pancreatectomy (LDP). Asvide

2016;3:331. Available online: http://www.asvide.com/ articles/1100

44. Zhou W, Lv R, Wang X, et al. Stapler vs suture closure of pancreatic remnant after distal pancreatectomy: a metaanalysis. Am J Surg 2010;200:529-36.

45. Diener MK, Seiler CM, Rossion I, et al. Efficacy of stapler versus hand-sewn closure after distal pancreatectomy (DISPACT): a randomised, controlled multicentre trial. Lancet 2011;377:1514-22.

46. Sepesi B, Moalem J, Galka E, et al. The influence of staple size on fistula formation following distal pancreatectomy. J Gastrointest Surg 2012;16:267-74.

47. Asbun HJ, Stauffer JA. Laparoscopic approach to distal and subtotal pancreatectomy: a clockwise technique. Surg Endosc 2011;25:2643-9.

48. Johnston FM, Cavataio A, Strasberg SM, et al. The effect of mesh reinforcement of a stapled transection line on the rate of pancreatic occlusion failure after distal pancreatectomy: review of a single institution's experience. HPB (Oxford) 2009;11:25-31.

49. Suzuki Y, Kuroda Y, Morita A, et al. Fibrin glue sealing for the prevention of pancreatic fistulas following distal pancreatectomy. Arch Surg 1995;130:952-5.

50. Velanovich $\mathrm{V}$. The use of tissue sealant to prevent fistula formation after laparoscopic distal pancreatectomy. Surg Endosc 2007;21:1222.

51. Oláh A, Issekutz A, Belágyi T, et al. Randomized clinical trial of techniques for closure of the pancreatic remnant following distal pancreatectomy. Br J Surg 2009;96:602-7.

52. Braga M, Ridolfi C, Balzano G, et al. Learning curve for laparoscopic distal pancreatectomy in a high-volume hospital. Updates Surg 2012;64:179-83.

53. Abu Hilal M, Hamdan M, Di Fabio F, et al. Laparoscopic versus open distal pancreatectomy: a clinical and costeffectiveness study. Surg Endosc 2012;26:1670-4.

54. Fox AM, Pitzul K, Bhojani F, et al. Comparison of outcomes and costs between laparoscopic distal pancreatectomy and open resection at a single center. Surg Endosc 2012;26:1220-30.

55. Rutz DR, Squires MH, Maithel SK, et al. Cost comparison analysis of open versus laparoscopic distal pancreatectomy. HPB (Oxford) 2014;16:907-14.

doi: 10.21037/jovs.2016.07.21

Cite this article as: Salman B, Yilmaz TU, Dikmen K, Kaplan M. Laparoscopic distal pancreatectomy. J Vis Surg 2016;2:141. 